\title{
Fragmentation of Leucine Enkephalin as a Function of Laser Fluence in a MALDI TOF-TOF
}

\author{
Jennifer M. Campbell and Marvin L. Vestal \\ Applied Biosystems, Framingham, Massachusetts, USA
}

\section{Paul S. Blank}

Laboratory of Cellular and Molecular Biology, National Institute of Child Health and Human Development, National Institutes of Health, Bethesda, Maryland, USA

\section{Stephen E. Stein}

Physical and Chemical Properties Division, National Institute of Standards and Technology, Gaithersburg, Maryland, USA

\section{Jonathan A. Epstein}

Unit on Biological Computation, National Institute of Child Health and Human Development, National Institutes of Health, Bethesda, Maryland, USA

\author{
Alfred L. Yergey \\ Laboratory of Cellular and Molecular Biology, National Institute of Child Health and Human Development, \\ National Institutes of Health, Bethesda, Maryland, USA
}

The effects of laser fluence on ion formation in MALDI were studied using a tandem TOF mass spectrometer with a Nd-YAG laser and $\alpha$-cyano hydrocinnamic acid matrix. Leucine enkephalin ionization and fragmentation were followed as a function of laser fluence ranging from the threshold of ion formation to the maximum available, that is, about $280-930 \mathrm{~mJ} / \mathrm{mm}^{2}$. The most notable finding was the appearance of immonium ions at fluence values close to threshold, increasing rapidly and then tapering in intensity with the appearance of typical backbone fragment ions. The data suggest the presence of two distinct environments for ion formation. One is associated with molecular desorption at low values of laser fluence that leads to extensive immonium ion formation. The second becomes dominant at higher fluences, is associated initially with backbone type fragments, but, at the highest values of fluence, progresses to immonium fragments. This second environment is suggestive of ion desorption from large pieces of material ablated from the surface. Arrhenius rate law considerations were used to estimate temperatures associated with the onset of these two processes. (J Am Soc Mass Spectrom 2007, 18, 607-616) (C 2007 American Society for Mass Spectrometry

$\mathrm{T}$ he fragmentation of protonated peptide ions to amino acid--specific sequence ions and the subsequent algorithm-based matching of the fragmentation constitute one of the primary processes enabling the mass spectrometric identification and characterization of proteins [1,2]. At the same time, understanding the fundamental features of the

Published online January 3, 2007

Certain commercial equipment, instruments, or materials are identified in this document. Such identification does not imply recommendation or endorsement by the National Institute of Standards and Technology, nor does it imply that the products identified are necessarily the best available for the purpose.

Address reprint requests to Dr. Alfred L. Yergey, National Institutes of Health, Building 10, Room 9D52, Bethesda, MD 20892. E-mail: aly@ helix.nih.gov processes behind the fragmentations will serve to increase both the scope of the problems addressable by tandem mass spectrometry (MS/MS) as well as the confidence in the answers obtained. In particular, investigations of the extent and type of fragmentation as a function of charge state, ion internal energy, attained by collision and/or formation mechanism or peptide sequence are vital. Such studies have been undertaken by various investigators [3-6] over a number of years, but nevertheless continue to be of central importance to the identification problem as shown by a recent conference organized to address such questions [7].

At the most fundamental level, the fragmentation of any gas-phase ion is a function of both the internal 
energy imparted to the ion and the length of time available for dissociation to occur $[8,9]$. Thus, the fragments observed in any mass spectrometer depend on the design of the mass spectrometer itselfthe ionization mechanism, the activation method, and the timescales for fragment formation and detection. Multiple groups have completed detailed analyses of the effects of each on fragmentation. The earliest studies focused on the fragmentations resulting from the relatively energetic radical cations obtained by electron ionization of derivatized peptides [10-12]. With the invention of fast atom bombardment (FAB) and the development of four-sector mass spectrometers, it became possible to study the effect of internal energy variation on fragmentation patterns $[3,13]$ arising from collisionally induced dissociations (CIDs). Simultaneously these studies made it possible to describe ion dissociation leading to the production of characteristic amino acid side-chain fragment ions. More recently, the remarkable successes of electrospray ionization (ESI) coupled with triple quadrupole or ion trap type mass analyzers has led to intense study of the low-energy fragmentations typically seen in these analyzers $[6,14]$. At the same time, more extensive fragmentation has been observed by Wysocki and coworkers [4] using surface-induced dissociation of electrosprayed ions. Other analyzers, such as the hybrid quadrupole-time of flight (Q-qTOF) instrument, also yield low-energy fragmentation, similar to that observed in triple quadrupoles and traps. Although insufficiently energetic to consistently yield complete fragmentations or to ever produce side-chain fragments, ion trap analyzers potentially allow sufficient time for the study of rearrangement reactions leading to sequence isomerization as reported by Glish and coworkers [15].

In principle matrix-assisted laser desorption ionization (MALDI) [16-18] could be as important an ionization method as ESI for protein and peptide characterization. To date, however, this method has been used mostly for peptide mass mapping rather than for the more informative peptide fragmentationbased approaches. Most likely the reason for this is the substantial effort required to undertake such studies in the absence of a tandem mass analyzer. Although post source decay (PSD) analysis [19] represents a pioneering way of simulating a tandem analyzer, the difficulty of reconstructing fragmentation spectra has precluded its being used for extensive peptide sequencing studies. The advent of true tandem TOF instruments $[20,21]$ has increased the interest in MALDI as an ionization method and has led to the development of new MALDI-based proteomics workflows, notably LC-MALDI [22]. One of these new analyzers, the MALDI TOF/TOF [20] has unique fragmentation patterns where the tandem spectra are a combination of the products of both high-energy CID and lower-energy PSD. The properties of both these types of fragmentation were previ- ously discussed elsewhere [23]. Detailed analyses of the properties of fragmentation as a function of collision parameters have been completed elsewhere [24]. The purpose of this work is to examine, in detail, the properties of fragmentation as a function of the energy used for ionization. To do this properly, one must examine in part the nature of the MALDI ionization process.

Ion formation in MALDI has been described as a result of three processes that follow the strong absorption of laser energy:

First, a controllable energy-transfer to the condensed-phase matrix-analyte mixture inducing a "uniform and soft desorption," second, a promotion of ionization by chemical reactions, and third, to generate favourable prerequisites "by isolating analyte molecules in the excess matrix" [25].

Fundamental studies of the MALDI process have addressed these three points from both the experimental and theoretical perspectives. Several groupsmost notably those of Karas, Hillenkamp, and, more recently, Driesewerd-have studied cluster ejection from the matrix, effects of sample morphology, plume dynamics, and initial velocities [25-28]. Internal energy coupled into the ions produced by these processes has been investigated by Zenobi and coworkers [29] and by Karas' group [25, 30]. From a theoretical perspective, molecular dynamics simulations of these processes have been carried out by groups led by Vertes [31-34], Garrison [35-38], and Knochenmuss [39]. In addition, Knochenmuss [40] developed an overall quantitative model for ion formation. Furthermore, there have been several studies conducted using a series of preformed "thermometer" ions that fragment in a manner well characterized by fundamental reaction rate theory [30, 33]. Most of these studies, however, have focused on the process of ionization itself, rather than the coupling of internal energy to the analyte during ionization. Thus, such studies ignore fragmentation post ionization. The experimental examination of the effect of laser fluence on ion fragmentation has also been investigated in dinucleotides [29], in protein complexes [41], and peptides [42, 43].

This paper presents the results of our preliminary investigations of peptide fragmentation and sets the stage for a systematic investigation of peptide fragmentations in MALDI. Although the overall aim of this and subsequent work is the optimization of peptide fragmentations produced by MALDI to enable more effective protein characterization, we have chosen leucine enkephalin (LeuEnk) for the initial study because of its extensive use by other investigators [14, 15, 44-48]. It is our intent to present a systematic study of the observed fragmentations and to characterize those observations within the context of the several existing models of the MALDI process. 


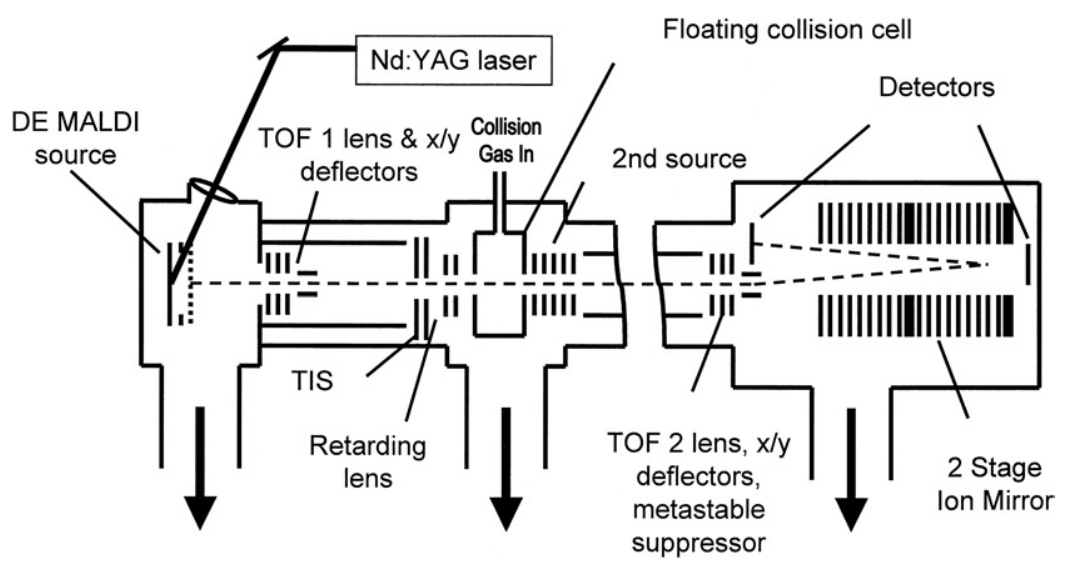

Figure 1. Schematic of the ABI 4700 Proteomics Analyzer MALDI TOF-TOF instrument. Note that TIS in schematic represents the "Timed Ion Selector" used for selection of precursor ions for MS/MS studies.

\section{Experimental}

\section{Mass Spectrometry}

An ABI 4700 Proteomics Analyzer MALDI TOF-TOF (MDS Sciex/ABI Proteomics, Concord, Ontario, Canada) was used in either MS or MS/MS mode for all measurements. The instrument is shown schematically in Figure 1. In MS, the accelerating voltage was lowered from the typical $20 \mathrm{kV}$ to $10 \mathrm{kV}$ to obtain optimal resolution of the low mass fragments. The grid was operated at $70 \%$ of the accelerating voltage and the time between the laser pulse striking the sample and the high-voltage extraction pulse was $400 \mathrm{~ns}$. The two lenses were used to provide optimal radial focusing, and the two-stage reflector was operated such that a resolution of 12,000 was attained. In reflector mode, only ions that are formed in the source will appear as focused features in the final spectrum. Without ramping the laser fluence, one would not be able to distinguish whether these features resulted from chemicals present in the sample or fragmentation resulting from the process of ionization. This mechanism of fragment formation is traditionally termed in source decay (ISD) [42, 43]. It should also be noted that ions that fragment after acceleration, but before the detector, have an energy defined by $\mathrm{E}_{\text {frag }}$ $=\mathrm{E}_{\text {prec }} \times\left(\mathrm{m}_{\text {frag }} / \mathrm{m}_{\text {prec }}\right)$ and would thus appear as unfocused metastable features in the spectrum. These are ignored in the analyses.

In MS/MS mode, it is useful to review instrument operation to understand the significance of the ions observed. In MS/MS mode in the TOF/TOF, ions are accelerated out of the first source and into a $40-\mathrm{cm}$-long field-free region. At the end of the field-free region, there is a timed ion selector (TIS) that prevents ions traveling at a velocity other than that of the precursor from entering the collision cell region. The collision cell region is operated at a floating potential and the nominal collision energy is defined by the potential difference between the accelerating voltage and the collision cell voltage. There is a deceleration stack that provides radial focusing for the ions entering the collision cell. The region defined as the collision cell is about $20 \mathrm{~cm}$ long, extending from the timed ion selector to the second source. The fragment ions, along with any remaining precursor ion, enter the second source simultaneously, and the extraction pulse of the second source is timed to pulse when the ions are about $4 \mathrm{~mm}$ into the source region. The pulse serves as the start time for the time to mass conversion of the fragment spectrum.

Because this study focuses on fragmentation, it is prudent to review in MS/MS mode, where fragmentation can occur. Ions fragmenting in the source (i.e., are analogous to those discussed above for reflector mode) will have a different velocity than that of the precursor and will be prohibited from entering the collision cell region, and thus the second source, by the timed ion selector. Similarly, ions that fragment during the flight time from the first source to the timed ion selector have the same velocity as that of the precursor, but have too low an energy to enter the collision cell region. Ions that fragment during the transit from the timed ion selector to the second source will arrive in the second source at the calculated extraction time for the precursor and will thus appear as focused fragment ions in the fragment spectra. Ions that fragment during the flight time from the second source to the detector will appear as unfocused metastable ions in the fragment spectrum.

For the MS/MS experiments completed here, an $8-\mathrm{kV}$ accelerating voltage, an $85 \%$ grid voltage, and a 400-ns delay between the laser and extraction highvoltage pulse was used in the first source. The system was operated at a nominal $1-\mathrm{kV}$ nominal collision energy, meaning that the potential offset between the source $(8 \mathrm{kV})$ and the collision cell $(7 \mathrm{kV})$ was $1 \mathrm{kV}$. No gas was added to the collision cell; thus fragments resulting from CID do not need to be considered. In the case of selecting the LeuEnk $\mathrm{MH}^{+}$as precursor, the time of flight from the first source to the timed ion selector is roughly $8 \mu \mathrm{s}$. It takes the same ions about $11 \mu$ s to travel from the timed ion selector to the second source, and 


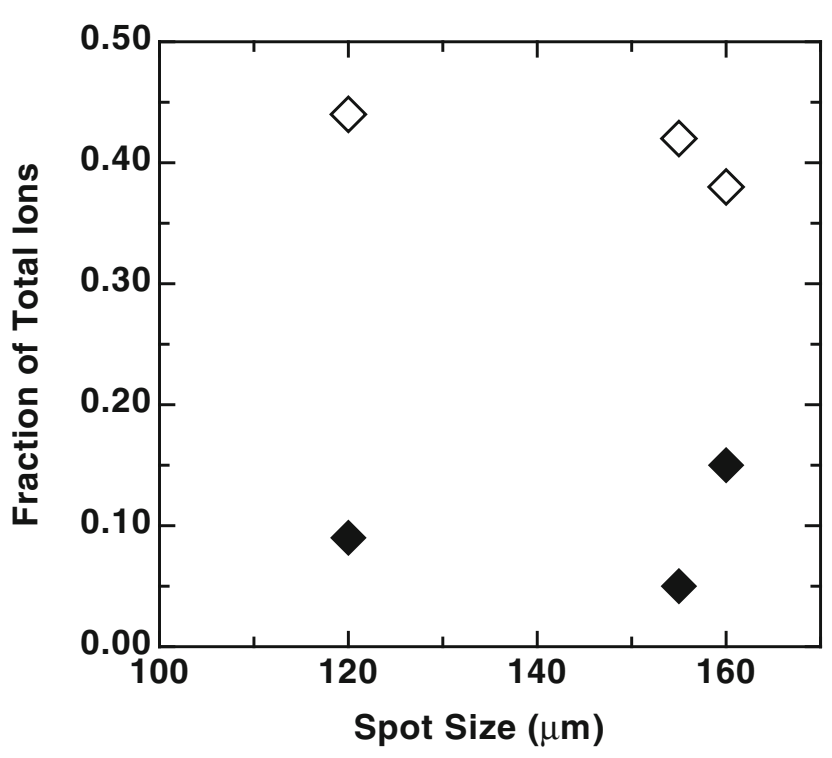

Figure 2. Extent of fragmentation as a function of spot size adjusted for comparable fluence. Data plotted as a fraction of total ions, where $\diamond=$ immonium ions and $\diamond=y$-series ions.

thus fragment ions observed in MS/MS mode are the result of ion dissociation taking between 8 and $20 \mu \mathrm{s}$. The second source was pulsed with an accelerating voltage of $15 \mathrm{kV}$ and a grid voltage of $7 \mathrm{kV}$.

The Nd:YAG laser (355-nm, 5-ns pulse width; LaserCompact, Moscow, Russia) was operated over a range of attenuator settings between 3900 and 6500 (arbitrary units), corresponding to the threshold for ion signal to maximum power. Laser power output was measured using a Molectron Energy Max 400 (Coherent, Inc. Santa Clara, CA) power meter and ranged from $285 \mu \mathrm{J} / \mathrm{mm}^{2}$ ("attenuator" setting of 3900) to $917 \mu \mathrm{J} / \mathrm{mm}^{2}$ ("attenuator" setting of 6500). Laser spot size on the sample plate was determined by ablating matrix from a sample plate coated only with a matrix solution until the underlying plate surface was exposed. Spot size was then estimated by microscopic comparison with a standard reference object. Results of these measurements were verified using a beam profiler. The majority of these experiments were completed with a laser spot size of $155 \mu \mathrm{m}$ diameter, but for comparison, spectra were obtained at spot sizes of 120 and $160 \mu \mathrm{m}$. It was determined that the three different spot sizes give rise to comparable degrees of fragmentation of protonated LeuEnk when fluence values were adjusted to comparable levels for each spot; this is shown in Figure 2.

LeuEnk samples were prepared as a $5 \mathrm{pmol} / \mu \mathrm{L}$ solution in a $0.1 \%$ trifluoroacetic acid (TFA) aqueous solvent. Matrix was a $5 \mathrm{mg} / \mathrm{mL}$ solution of recrystallized $\alpha$-cyano hydrocinnamic acid (CHCA) in a 1:1 acetonitrile: $0.1 \%$ TFA solution made $10 \mathrm{mM}$ in $\left(\mathrm{NH}_{3}\right)_{2} \mathrm{H}_{2} \mathrm{PO}_{4}[49,50]$. A 20:1 matrix:sample mixture was made, applied to the sample plate as $1 \mu \mathrm{L}$ drops, and allowed to air dry. A given sample spot was used to generate only a single 5000 laser shot spectrum, thus ensuring each spectrum was generated from surfaces of a similar physical state with no possibility of sample depletion. Laser attenuation was stepped incrementally over the range described above under automated control software. The absence of signal saturation in the averaged spectra, particularly at intermediate attenuator settings, was accomplished by instrument control software (single shot protection feature) to reject single shot spectra with signal intensities $>8.5 \times 10^{4}$ counts from being averaged into the final spectrum. In MS/MS mode no metastable suppressor was used, and thus precursor and fragment areas reflect abundances resulting from fragmentation and instrument transmission.

\section{Materials}

Acetonitrile (HPLC grade), TFA, and $\left(\mathrm{NH}_{3}\right)_{2} \mathrm{H}_{2} \mathrm{PO}_{4}$ (reagent grade) were used as received from the supplier (Aldrich, Milwaukee, WI); CHCA (Aldrich) was recrystallized before use. LeuEnk (Sigma, St. Louis, MO) was used as received.

\section{Data Analysis}

Individual spectra were copied from the default database format of the 4700 Version 2.0 Console software into individual files accessible to a freestanding version of the ABI Data Explorer ${ }^{\mathrm{TM}}$ software. Using a Perl script written to execute a batch task, spectra were calibrated externally against a LeuEnk spectrum obtained close to the onset of ionization. Spectra were calibrated in MS mode using $\mathrm{m} / \mathrm{z}$ ratios of LeuEnk $\mathrm{MH}^{+}$and the matrix monomer and dimer ions and in MS/MS mode by using the $\mathrm{MH}^{+}$and $m / z 136$ fragment ion. Tables of $\mathrm{m} / \mathrm{z}$-intensity pairs were generated for ions exceeding a signal-to noise ratio $(\mathrm{S} / \mathrm{N})$ of 5 . To account for differences in resolution as a function of $\mathrm{m} / \mathrm{z}$ in both MS and MS/MS modes, the area of the C12 isotope peak was used as the metric of ion abundance. These tables were then written in ASCII format under control of the same Perl script; the tables were subsequently parsed using a separate Perl script into summary tables containing intensity information for LeuEnk fragment ions as a function of attenuator setting/laser fluence. All subsequent data analysis was carried out using these final

Table 1. Fragments of leucine enkephalin (YGGFL, $\mathrm{MH}^{+}=$ 556.28)

\begin{tabular}{|c|c|c|c|c|}
\hline \multirow[b]{2}{*}{ Ion Series } & \multicolumn{4}{|c|}{ Fragment } \\
\hline & 1 & 2 & 3 & 4 \\
\hline y & 132.10 & 279.17 & 336.19 & 393.21 \\
\hline b & 164.07 & 221.09 & 278.11 & 425.18 \\
\hline a & $136.08^{a}$ & 193.10 & 250.12 & 397.19 \\
\hline Internal fragments: & \multirow{2}{*}{\multicolumn{4}{|c|}{$\begin{array}{l}115.05 \text { (GG), } 205.10 \text { (GF), } 262.12 \text { (GGF) } \\
86.10(\mathrm{~L}), 120.08(\mathrm{~F}), 136.08(\mathrm{Y})\end{array}$}} \\
\hline Immonium & & & & \\
\hline
\end{tabular}

antensity of $\mathrm{m} / \mathrm{z} 136.08$ is taken as immonium $(\mathrm{Y})$ rather than $\mathrm{a}_{1}$. blmmonium of $\mathrm{G}(\mathrm{m} / \mathrm{z} 30)$ is below scan limit of the experiment. 

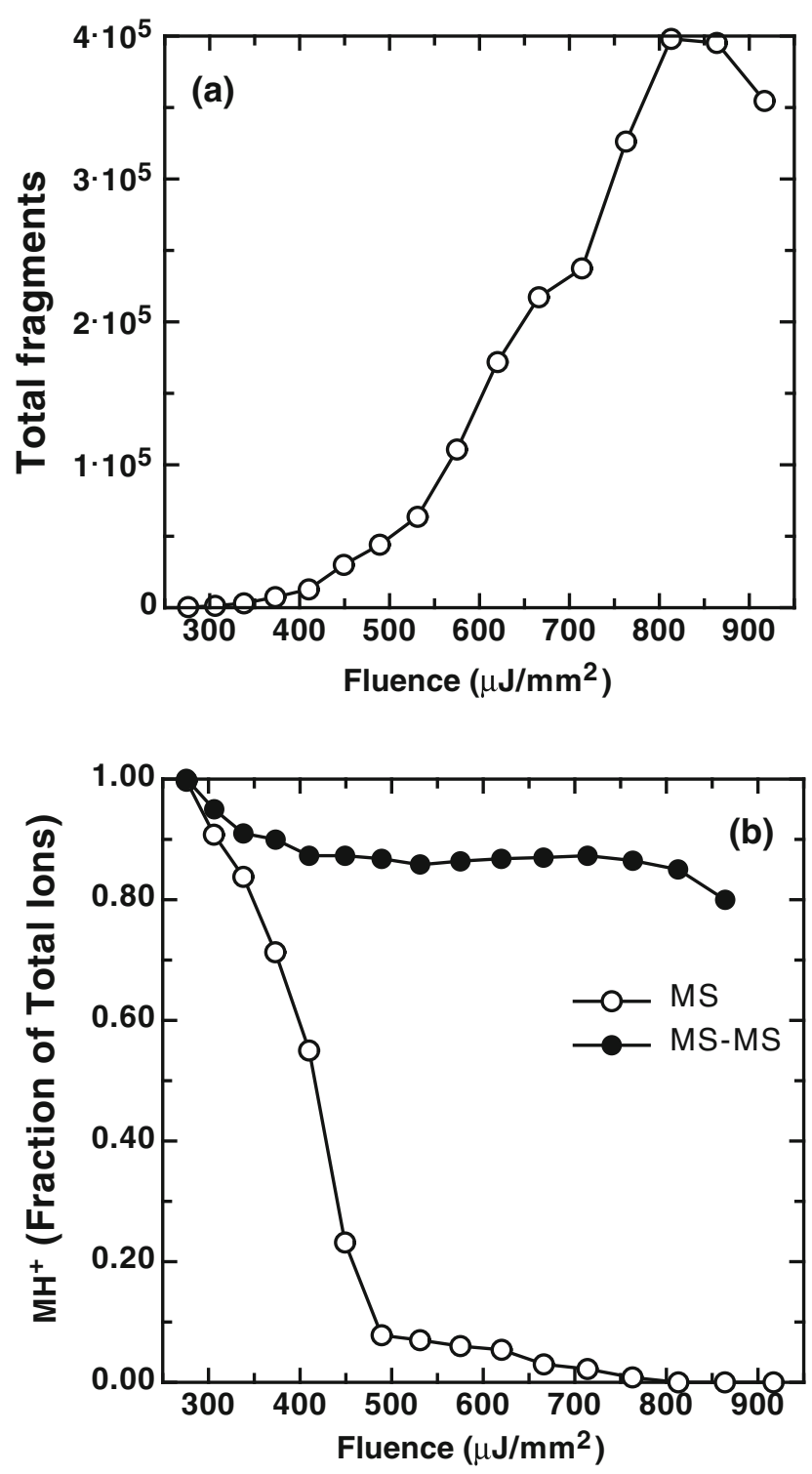

Figure 3. (a) Total fragment ion signal versus laser fluence plotted over operating fluence range. (b) LeuEnk $\mathrm{MH}^{+}$signal intensity versus laser fluence plotted as a fraction of total ion signal normalized at each laser fluence level, for MS $(\bigcirc)$ and MS/MS (•) modes.

tables. The masses and ion types used for this parsing are listed in Table 1.

\section{Results and Discussion}

\section{Total Fragments-Protonated Parent and Precursor}

The overall behavior of LeuEnk as a function of laser fluence is illustrated in Figure 3 with total ion current shown in the upper panel and $\mathrm{MH}^{+}$intensity, as a fraction of total fragment ion current, for both MS and MS/MS modes shown in the lower panel. Figure 3B shows that in MS mode the $\mathrm{MH}^{+}$ion intensity rapidly diminishes as fluence increases above the threshold for ion production, up to about $480 \mu \mathrm{J} / \mathrm{mm}^{2}$. After this point the intensity continues to diminish, but at a considerably slower rate until it disappears entirely just above $800 \mu \mathrm{J} / \mathrm{mm}^{2}$. In MS/MS mode, on the other hand, the protonated parent ion remains the base peak of spectra over the whole range of laser fluence. It is clear from Figure 3 that there is an association between laser fluence and ion fragmentation and it is also apparent that the extent of the fragmentation is associated with differences in the time at which the $\mathrm{MH}^{+}$ion is sampled, that is, MS versus MS/MS. We believe, in light of the discussion below, that this behavior is internally consistent.

\section{Fragmentation}

Laser fluence-induced fragmentation processes in MS mode are shown in more detail in the breakdown curves of Figure 4. Because all fragment ions observed in MS mode must be formed before exiting the source, these must be considered prompt fragments. Peak areas resulting from each of five different types of dissociations are summed for the figure, that is, the $\mathrm{y}_{-}^{-}, \mathrm{b}-$, and a-series ions, internal fragments, and immonium ions. It can be seen in this figure that, whereas immonium ion production quickly becomes the dominant process, at the very onset of fragmentation immonium ion formation appears to be preceded by backbone fragmentations of both $y$ - and b-series ions. In terms of the immonium ions observed, both Phe-, $\mathrm{m} / \mathrm{z} 120$, and Tyr-immonium, $m / z$ 136, ions contribute equally to this

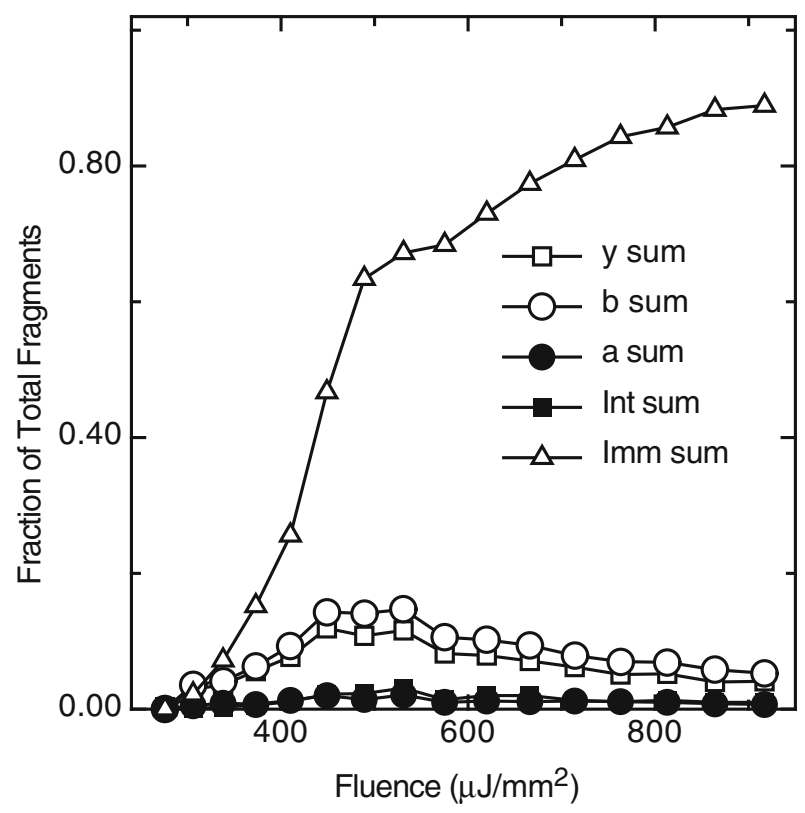

Figure 4. Summed fragment ion signal intensity in MS mode versus laser fluence plotted as a fraction of total ion signal normalized at each laser fluence level. Five fragment ion series shown as normalized sums: $\triangle$, immonium; $\bigcirc$, b-series; $\bullet$, a-series; $\square$, y-series; $\mathbf{\square}$, internal ions. 

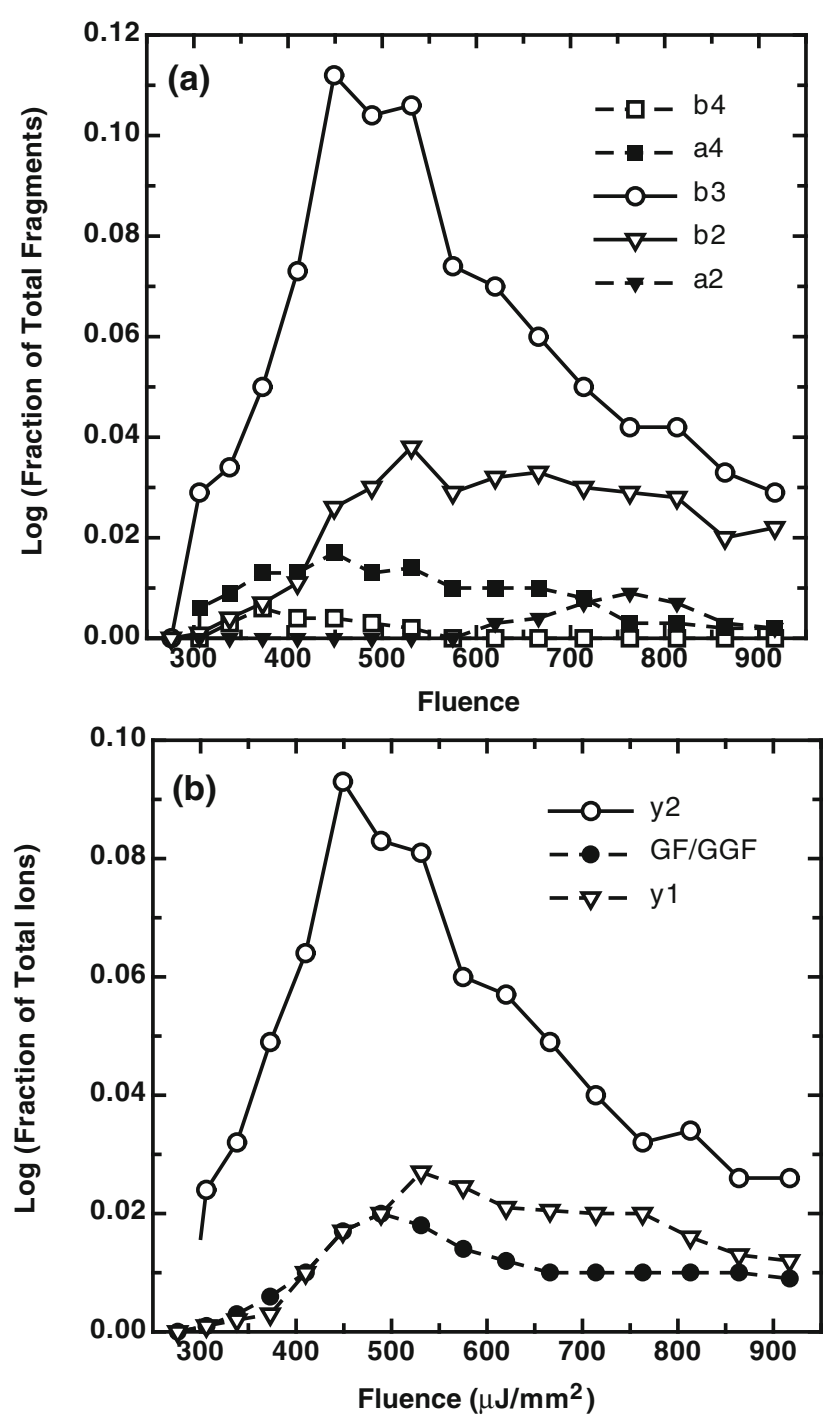

Figure 5. Fragment ion signal intensity for consecutive fragmentations in MS mode versus laser fluence, as a fraction of total ion signal normalized at each laser fluence level. (a) N-terminal ions: $\square\left(\mathrm{b}_{4}\right), \boldsymbol{\square}\left(\mathrm{a}_{4}\right), \bigcirc\left(\mathrm{b}_{3}\right), \nabla\left(\mathrm{b}_{2}\right), \boldsymbol{\nabla}\left(\mathrm{a}_{2}\right)$. (b) C-terminal ions: $\bigcirc\left(\mathrm{y}_{2}\right)$, $(\mathrm{GF}$ and $\mathrm{GGF}), \nabla\left(\mathrm{y}_{1}\right)$.

curve over the entire range measured, although there are no contributions observed from either the Gly- or Leu-immonium ions at $\mathrm{m} / \mathrm{z} 30$ and 86 , respectively. Note that, although the $\mathrm{m} / \mathrm{z} 136$ ion is isobaric with the $\mathrm{a}_{1}$ fragment, we assign its origin to the immonium series because of its strong resemblance between the breakdown curves for $m / z 120$ and 136 . The threshold behavior of these processes is not inconsistent with those predicted by Knochemmuss [40] for similar laser spot sizes considering that our measurements used CHCA matrix rather than the $\mathrm{DHB}$ of the calculations.

The second intriguing characteristic of Figure 4 is the behavior of the C-and N-terminal fragment ions. Figure 4 suggests the occurrence of something other than a simple response to increasing laser fluence for these two ion series. That this is indeed the case is shown more clearly in Figure 5 where details of $\mathrm{N}$-terminal fragments are shown in the upper panel and C-terminal fragments in the lower. Figure 5a shows a progression from higher to lower mass members of the two series as a function of laser fluence. This progression is consistent with a series of consecutive fragmentations. Harrison [6] and Alexander and Boyd [14] previously demonstrated such consecutive fragmentations for LeuEnk, using electrospray ionization and in-source CID, but to our knowledge, such behavior has not been previously demonstrated in MALDI.

If the energy required to yield a particular fragment is indeed a function of laser fluence, then the succession of maxima in Figure 5 should yield an energy-dependent ion series. Based on the observed progression of maxima, the energy-dependent series for the N-terminal fragments is: $b_{4}<a_{4}<b_{3}<\left[a_{3}\right]<$ $b_{2}<a_{2}$. There was no signal seen for the $a_{3}$ ion and, interestingly, it was absent from the series reported by Harrison [6]. There was also no signal observed for the $b_{1}$ ion either and, if the $a_{1}$ is truly present, its existence was masked by the immonium ion as noted above.

An analogous pattern was observed for the Cterminal series, the $\mathrm{y}$ ions and internal fragments, shown in Figure $5 \mathrm{~b}$. The $\mathrm{GF}^{+}$and $\mathrm{GGF}^{+}$internal fragments overlap in their behavior and for this reason only the $\mathrm{GF}^{+}$signal is plotted. No intensities that can be associated with the $\mathrm{GG}^{+}$internal ion were observed. The C-terminal ions observed, in order of increasing energy requirements, are: $\mathrm{y}_{2}<\mathrm{GG}^{+} /$ $\mathrm{GGF}^{+}<\mathrm{y}_{1}$. A similar progression of energy requirements was reported by Harrison [6] over ESI source cone voltages in the range of 44-62 V. The presence of the $\mathrm{GF}^{+}$internal fragment was reported by only two other groups, Alexander and Boyd [14] in a CID spectrum with $\mathrm{E}_{\mathrm{LAB}}=70 \mathrm{eV}$ with $90 \%$ attenuation of $\mathrm{MH}^{+}$using a sector instrument and Wysocki and coworkers [44] under thermal degradation conditions for which the ions were at temperatures between 658 and $679 \mathrm{~K}$. In general other studies appear to have been undertaken under CID [48, 51] or thermal conditions [47], which were insufficiently energetic to allow decompositions to the extent of producing either these internal fragments or immonium ions.

It is interesting to note in Figure 5a that the intensity of any a-series ions observed is roughly tenfold lower than the b-series ion from which it derives or to which it leads. Further inspection of Figure 5 shows that the most intense ions are the complementary pair, $b_{3}$ and $\mathrm{y}_{2}$, and that their maxima occur at the same laser fluence. Taken together, these data make a clear case for the presence of a series of energy-dependent fragmentations that occur within the ion source of the instrument.

Consideration of the fragmentation observed in MS/MS mode, as seen in Figure 6, reveals an appreciably less complex pattern than that seen in MS mode. Neither internal nor immonium ion fragments are seen over the entire range of laser fluence in MS/MS mode and only a limited number of $\mathrm{C}$ - and $\mathrm{N}$-terminal ions are observed. Although the total $\mathrm{C}$ - and $\mathrm{N}$-terminal 


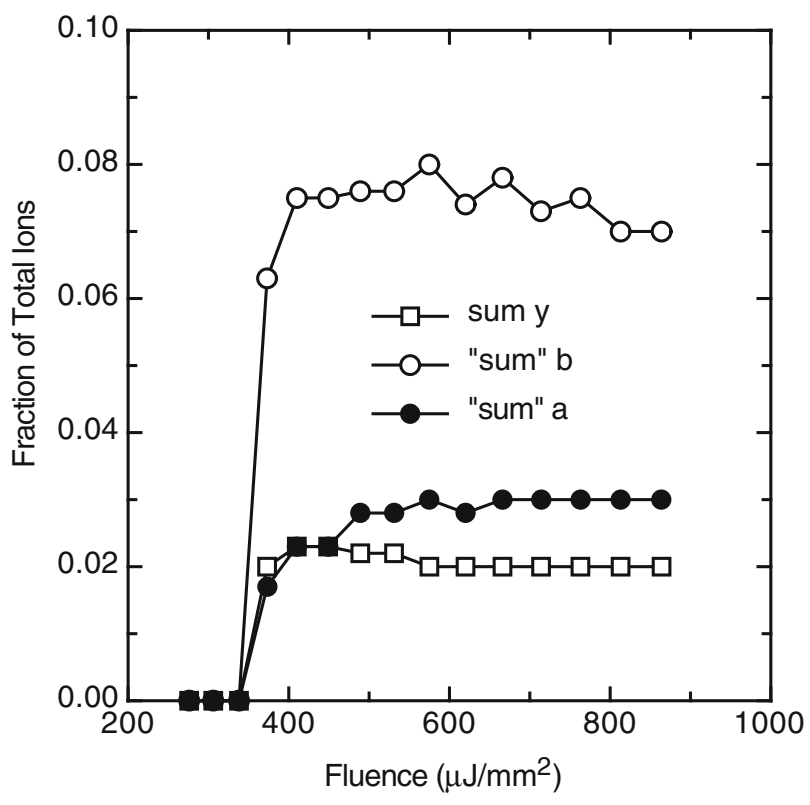

Figure 6. Fragment ion signal intensity for consecutive fragmentations in MS/MS mode versus laser fluence, as a fraction of total ion signal normalized at each laser fluence level: $\square$ (sum of y-series), $\bigcirc$ (sum of b-series), $\bullet$ (sum of a-series).

fragments are of comparable relative intensity to each other as those observed in MS mode, any clear indication of a series of consecutive reactions is absent in MS/MS; those fragments that are observed are present at all values of fluence. Furthermore, the onset of these fragment signals in MS/MS occurs at appreciably higher fluence levels than in MS.

A general interpretation of the overall behavior of LeuEnk fragmentation shown in Figures 4-6 suggests several significant points. There appear to be two broad classes of reactions that occur within the $1-\mu$ s interval accessible in MS mode. These classes correspond, first, to a rapid and extensive coupling of energy into the molecule leading to extensive fragmentations as suggested by the presence of immonium fragments close to the onset of ionization; this is termed Process 1. This process leads to extensive fragmentation resulting in rapidly increasing levels of immonium ions up to a laser fluence of about $500 \mu \mathrm{J} / \mathrm{mm}^{2}$. Above this value of fluence, the rapid production of immonium ions versus fluence appears to become slower.

The sequence ions seen in MS, and the similar ions observed in MS/MS, as shown in Figures 4-6, suggest the presence of a second, discrete, energy distribution by which smaller amounts of energy appear to be coupled into the molecule; this will be referred to as Process 2. This second class of reaction leads to a series of fragmentations that proceed as a series of consecutive reactions in which the product of each reaction gains the thermal energy necessary to serve as the precursor of the next reaction. These consecutive fragmentations suggest the presence of a second ion fragmentation process. One might speculate that these arise as a result of a somewhat separable energy distribution in the plume of ions and neutral molecules formed by the laser irradiation. If distribution of ion energies associated with Process 2 were indeed present, then it might also serve as the basis for the fragmentation seen in MS/MS mode. That is, in MS/MS fragments would be those arising from decompositions of $\mathrm{MH}^{+}$ions in the 8- to 20- $\mu$ s interval, which had insufficient internal energy for prompt fragmentation, yet sufficient to fragment in the longer, MS/MS time interval. The second set of fragmentations seen in MS and all of those seen in MS/MS suggest the presence of an energy distribution that is somewhat distinct from the first one described above within the plume of ions generated from the sample.

Figure 7 is an attempt to represent the second group of decompositions in MS mode (i.e., those associated with Process 2). The figure was generated by subtracting the immonium ion intensity at $480 \mu \mathrm{J} / \mathrm{mm}^{2}$ from the total immonium ion intensity and replotting Figure 4 using only those immonium ion intensities greater than zero. Figure 7 clearly shows that the second group of prompt fragmentations proceeds from backbone processes to immonium ion formation. Note, the assumption behind this figure is that the first process continues over the entire range of fluences studied but contributes relatively less than the fragments of the second set of processes above a fluence of $500 \mu \mathrm{J} / \mathrm{mm}^{2}$.

There are several possible explanations that might account for the observations summarized in Figures $4-7$. Before presenting them it is critical to note that our aim in this paper is to present what is clearly a novel set of observations-the fragmentation of an easily frag-

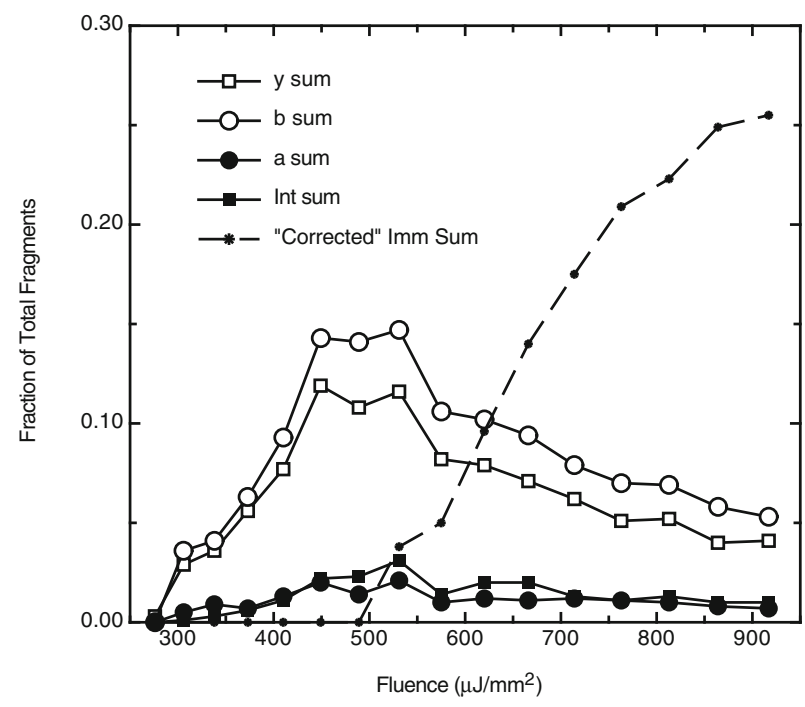

Figure 7. Summed fragment ion signal intensity in MS mode, as a fraction of total ion signal normalized at each laser fluence level versus laser fluence plotted for five fragment ion series. Immonium signal intensity "corrected" from data of Figure 4 to emphasize terminal consecutive reactions of Process 2 described in text: $\square$ (sum of y-series), $\bigcirc$ (sum of b-series), $\bullet$ (sum of a-series), (Internal ions), ("Corrected" sum of immonium ions). 
mented peptide under MALDI conditions where the laser fluence is increased from threshold to the maximum available for the instrument. What we intend to provide is the simplest physical model to account for the observations in our extensive experimental study. It is not our intention to formulate a new theoretical model for MALDI. Furthermore, our hope is that the explanation we provide is model independent and therefore neither supports nor negates any of the existing MALDI models.

To summarize our interpretation of the data, we suggest that these data support the presence of two classes of processes: the one beginning just above the threshold for ionization and the other beginning at about $480 \mu \mathrm{J} / \mathrm{mm}^{2}$. Both processes lead to progressive fragmentation of the $\mathrm{MH}^{+}$ion, but the first appears to couple energy into the protonated parent much more quickly than the second. As a result, the first process shows a rapid dominance of the immonium ion intensity in the $\mathrm{MH}^{+}$breakdown curves. On the other hand, the second process, although ultimately reaching the same point (i.e., domination by immonium ion production) proceeds in a manner in which energy is coupled into the $\mathrm{MH}^{+}$more slowly than the first. In very simple terms, in this system of analyte and matrix, the first process leads to immonium ions being dominant at fluences $>350 \mu \mathrm{J} / \mathrm{mm}^{2}$, whereas the second requires fluences $>600 \mu \mathrm{J} / \mathrm{mm}^{2}$. We also suggest that the first process is strongly associated with the duration of the laser pulse and the second is more strongly associated with the time required for the ion extractions from the first source to occur. That is, we suggest that the timing for ion production by the first process is about the laser flash duration, $5 \times 10^{-9} \mathrm{~s}$, and the timing for ion production by the second process is about $1 \times 10^{-6} \mathrm{~s}$.

The simple physical model that has just been described might be reconciled with two somewhat different theoretical models. In the first, a molecular dynamics simulation developed by Garrison and coworkers [36], there is a progression with increasing laser fluence from the initial desorption of individual molecules through chunks of ablated material. These ablated chunks ultimately progress, with increasing fluence, to smaller pieces and finally essentially individual molecules. Such a progression might account for our observations if the initial individual molecules were desorbed with high internal energies, which in our case would lead to the rapid production of immonium ions as seen in Figure 4. As the chunks ablated, one might expect ions to be produced with lower internal energies by desorption from the overall somewhat cooler chunks. This would lead to the observed production of backbone fragments that ultimately progress to the immonium ions as shown in Figure 7. However, a recent paper by Knochenmuss and Zhigilei [39] shows that this simple interpretation of the molecular dynamics simulation may be incomplete. Their work predicts that the initial individually emitted molecules are no hotter than the clusters. They furthermore show that the
Table 2. Parameter values ${ }^{\mathrm{a}}$

\begin{tabular}{lcc}
\hline & $\mathrm{A}\left(\mathrm{s}^{-1}\right)$ & $\mathrm{E}^{\ddagger}(\mathrm{kcal} / \mathrm{mol})$ \\
\hline \hline Estimate 1 & $10^{16}$ & 37 \\
Estimate 2 & $10^{15}$ & 25 \\
\hline
\end{tabular}

aparameter values are from Mautner et al. (44) and Stein (54).

cluster temperatures do not differ from that of the surrounding gas.

The molecular dynamics simulations, however, might still provide a means of explaining the origins of the first group of processes and thereby account for our observations. As proposed in two recent papers and a review by Knochenmuss [39, 52, 53], laser irradiation of the MALDI sample induces charging of the upper layers of the sample through the migration of mobile free electrons from the surface. The migration yields a charged surface of the sample that could in turn lead to the ejection of protonated ions from the surface. These ejected ions will carry internal energies associated with the temperature of the heated surface and, because of low molecular densities above the surface, would not thermalize, but rather fragment in a manner consistent with our observations. Although this process becomes masked at higher fluences, it should persist over the entire range studied, in a manner consistent with Figure 7.

On the other hand, at high fluences the relatively dense plume leads to a near equilibrium state that favors proton transfers from matrix to analyte. As the fluence increases beyond $480 \mu \mathrm{J} / \mathrm{mm}^{2}$, the increasing plume density leads to higher numbers of ion-molecule collisions, approaching a high-pressure limit and quasiequilibrium conditions. Under these circumstances, energy deposition into the LeuEnk $\mathrm{MH}^{+}$is limited by collisions with the plume gases and increases only with plume temperature/laser fluence giving rise to the observed sequence of fragments, dominated ultimately by immonium ions.

\section{Energetic Considerations}

The temperatures at which the two classes of processes occur can be estimated using an approach based on the Arrhenius rate law. As a starting point, we assume that all of the prompt fragmentation reactions, those observed in MS mode, occur near the "high-pressure" regime for unimolecular reactions. We make this assumption initially for reasons discussed below. The high-pressure limit is generally assumed to exist in the laser-induced plume of ions and neutrals of the size (number of oscillators) of concern in this work. Although not likely a classical equilibrium state, we assume that we can derive an effective temperature in the regime of interest. To estimate temperature, we equate the unimolecular reaction rate constant (k), describing the loss of $\mathrm{MH}^{+}$intensity with the Arrhenius rate constant expression as shown in the following equation: 
Table 3. Results

\begin{tabular}{lccc}
\hline & $\mathrm{t}(\mathrm{s})$ & Extent $\left(1-\left[\mathrm{MH}^{+} / \mathrm{MH}_{0}\right]\right)$ & Estimate 2 \\
\hline \hline High-pressure limit & $1 \times 10^{-6}$ & 0.02 & $694 \mathrm{~K}$ \\
Process 1 only & $1 \times 10^{-6}$ & 0.16 & $755 \mathrm{~K}$ \\
& $5 \times 10^{-9}$ & 0.16 & $562 \mathrm{~K}$ \\
\hline
\end{tabular}

$$
\mathrm{k}=(1 / \mathrm{t}) \ln \left(\mathrm{MH}_{0}^{+} / \mathrm{MH}^{+}\right)=\mathrm{A} \exp \left(\mathrm{E}^{\ddagger} / \mathrm{kT}\right)
$$

where $\mathrm{E}^{\ddagger}$ and $\mathrm{A}$ are the activation and frequency factor, respectively; $\mathbf{k}$ is the Boltzmann constant; $\mathrm{T}$ is the absolute temperature; and $t$ is the duration of the reaction. The rate constant can be eliminated from eq. 1 and the resulting expression solved for $\mathrm{T}$, the temperature at which an observed extent of reaction will be achieved for particular values of $\mathrm{A}$ and $\mathrm{E}^{\ddagger}$.

The temperatures to be evaluated are those in what we propose as two different reaction regimes. Initially we will assume that both groups of reactions occur in the high pressures that are likely associated with the higher laser fluences. Furthermore, we will initially assume that both reactions occur in the longer time frame being considered: $1 \times 10^{-6} \mathrm{~s}$, the time necessary to extract the ions from the source. For the initial temperature estimates we will differentiate the two groups of reaction from one another by their reaction extent. We assume that the process leading to extensive fragmentation, essentially breaking sufficient bonds to yield only immonium ions, breaks about $16 \%$ of the bonds in the peptide. This reaction extent must occur in two regions of the observed data-that is, in the region between threshold and the break seen in Figure 4 (below about $480 \mu \mathrm{J} / \mathrm{mm}^{2}$ ) and at fluences above about $600 \mu \mathrm{J} / \mathrm{mm}^{2}$. On the other hand, the beginnings of the second process, at about $480 \mu \mathrm{J} / \mathrm{mm}^{2}$, would appear to involve a lower extent of reaction, breaking only the bonds necessary to form a single $\mathrm{y}$ - or b-series ion, or about $2-3 \%$ of the peptide bonds. The Arrhenius parameters used for the two estimates are given in Table 2. Because Arrhenius parameters for the dissociation of LeuEnk are not accurately known, we use high and low estimates given in this table in an effort to bracket temperatures. The Arrhenius parameters for the first estimate were taken from the thermal degradation measurement of Mautner et al. [44], which showed the presence of immonium ions and those for second from typical peptide dissociations [54]. Results of the calculations for the two sets of Arrhenius parameters, a 1- $\mu \mathrm{s}$ reaction time and the two different extents of reaction, are shown in the upper part of Table 3.

As expected, Table 3 indicates a higher temperature is required for a greater extent of reaction. The temperatures calculated for the 2\% reaction extent, 513-694 K, are rather close to those observed in blackbody infrared radiative dissociation (BIRD) work [47], in which backbone fragmentation ions were found to predominate the spectra. They are not inconsistent with the temperature for the onset of ion formation predicted by
Knochenmuss [55]. Immonium ion production becomes dominant in what we have termed Process 2 at a $16 \%$ reaction extent corresponding to a fluence of about 600 $\mu \mathrm{J} / \mathrm{mm}^{2}$. We estimate the temperature necessary to induce these fragmentations to be in the range $562-755 \mathrm{~K}$.

To produce immonium ions by what we have termed Process 1, we speculate that the bond ruptures occur in a shorter time frame than those that are simply limited by the extraction pulse length; we suggest a time frame of 5-ns laser pulse duration. Recognizing that such processes are not likely to be strictly amenable to estimation using the approach taken in eq. 1, we nevertheless use it here. We assume the same values of activation energy and preexponential factors and reaction extent, but simply shorten the time for the reaction to proceed. The results of this calculation are in the last line of Table 3; they show a temperature range of $736-960 \mathrm{~K}$. The lower end of this range is in reasonable agreement with the immonium ion formation observed in thermal degradation measurements by Wysocki's group [44], between 658 and $679 \mathrm{~K}$.

In terms of the MS/MS fragmentations that are observed, we suggest that these ions result from processes arising from the low-energy portion of a thermal distribution - that is, ions that do not fragment in the source, but do so when given the time frame available in MS/MS. Figure 6 shows that the MS/MS fragments are the product of the lowest energy pathways available to the $\mathrm{MH}^{+}$and are not observed until appreciably above the onset of the backbone type fragments shown in Figure 5. From this we conclude that the fragments seen in MS/MS arise only from the low-energy portion of the distribution generated by the chunk ablation process of the laser.

\section{Conclusions}

Leucine enkephalin has been shown to undergo two regimes of ion formation as a function of MALDI laser fluence. These two regimes correspond to two different ion-formation environments that occur within the MALDI plume at different levels of fluence. The first is associated with molecular desorption from the surface even at relatively low fluence values, but leads to virtually complete fragmentation within very short times. The second process is associated with the evaporation of ions from large pieces of material ablated from the sample surface, occurs at longer times, and is associated, initially at least, with consecutive backbone fragmentations. There is a clear point of crossover between these two different processes. Although 
leucine enkephalin is a rather small peptide given to extensive fragmentation, nevertheless these initial studies appear to give significant experimentally based insights into the processes that occur in MALDI ion formation.

\section{Acknowledgments}

This research was supported, in part, by the Intramural Research Program of the National Institute of Child Health and Human Development.

\section{References}

1. Ferguson, P.; Smith, R. Proteome Analysis by Mass Spectrometry. Annu. Rev. Biophys. Biomol. Struct. 2003, 32, 399-424.

2. Steen, H.; Mann, M. The ABC's (and XYZ's) of Peptide Sequencing. Nat. Rev. Mol. Cell. Biol. 2004, 5, 699-711.

3. Johnson, R.; Martin, S.; Biemann, K. Collision-induced Fragmentation of $(\mathrm{M}+\mathrm{I}-1)+$ Ions of Peptides. Side Chain Specific Sequence Ions. Int. J. Mass Spectrom. Ion Process. 1988, 86, 137-154.

4. Jones, J.; Dong, A.; Somogyi, A.; Wysocki, V. Sequence Dependence of Peptide Fragmentation Efficiency Curves Determined by Electrospray Ionization/Surface-induced Dissociation Mass Spectrometry. J. Am. Chem. Soc. 1994, 116, 8368-8369.

5. Vachet, R.; Winders, A.; Glish, G. Correlation of Kinetic Energy Losses in High-Energy Collision-induced Dissociation with Observed Peptide Product Ions. Anal. Chem. 1996, 68, 522-526.

6. Harrison, A. Energy-resolved Mass Spectrometry: A Comparison of Quadrupole Cell and Cone-voltage Collision-induced Dissociation. Rapid Commun. Mass Spectrom. 1999, 13, 1663-1670.

7. Geer, L.; Yergey, A.; Stein, S.; Nesvizhskii, A.; Markey, S.; Kowalak, J.; Epstein, J. NIH/NIST/ISB Peptide Fragmentation and Identification Workshop, April 8-9, 2004.

8. Benson, S. Thermodynamical Kinetics; McGraw-Hill: New York, 1960

9. Forst, W. Theory of Unimolecular Reactions; Academic Press: New York, 1973.

10. Biemann, K.; Gapp, F.; Seibl, J. Application of Mass Spectrometry to Structure Problems. I. Amino Acid Sequence in Peptides. J. Am. Chem. Soc. $1960,81,2274-2275$.

11. Stenhagen, E. Massenspektrometrie als Hilfsmittel bei der Strukturbestimmung Organischer Verbindungen, Besonders bei Lipieden und Peptiden. Fresenius' J. Anal. Chem. 1961, 181, 462-480.

12. Biemann, K. Mass Spectrometry: Organic Chemical Applications; McGraw-Hill: New York, 1962, p 370.

13. Bordoli, R.; Bateman, R. The Effect of Collision Energy, Target Gas and Target Gas Purity on the High Energy Collision Induced Product Ion Spectrum of Renin Substrate. Int. J. Mass Spectrom. Ion Process. 1992, 122, 243-254.

14. Alexander, A.; Boyd, R. Experimental Investigations of Factors Controlling the Collision Induced Dissociation Spectra of Peptide Ions in a Tandem Hybrid Mass Spectrometer. I. Leucine Enkephalin. Int. J. Mass Spectrom. Ion Process. 1989, 90, 211-240.

15. Vachet, R.; Asam, M.; Glish, G. Secondary Interactions Affecting the Dissociation Patterns of Arginine-Containing Peptide Ions. J. Am. Chem. Soc. 1996, 118, 6252-6256.

16. Karas, M.; Hillenkamp, F. Laser Desorption Ionization of Proteins with Molecular Masses Exceeding 10,000 Daltons. Anal. Chem. 1988, 60, 2299-2301.

17. Tanaka, K.; Waki, H.; Ido, Y.; Akita, S.; Yoshida, Y.; Yoshida, T. Protein and Polymer Analysis up to $m / z 100,000$ by Laser Ionization Time-ofFlight Mass Spectrometry. Rapid Commun. Mass Spectrom. 1988, 2, 151-153.

18. Hillenkamp, F.; Karas, M.; Beavis, R.; Chait, B. Matrix-assisted Laser Desorption of Biopolymers. Anal. Chem. 1991, 63, 1193A-1203A

19. Spengler, B. Post-source Decay Analysis in Matrix-assisted Laser Desorption/Ionization Mass Spectrometry of Biomolecules. J. Mass Spectrom. 1997, 32, 1019-1036.

20. Medzihradszky, K.; Campbell, J.; Baldwin, M.; Juhasz, P.; Vestal, M.; Burlingame, A. The Characteristics of Peptide Collision-induced Dissociation Using a High-performance MALDI-TOF/TOF Tandem Mass Spectrometer. Anal. Chem. 2000, 72, 552-558

21. Shevchenko, A.; Loboda, A.; Shevchenko, A.; Ens, W.; Standing, K. MALDI Quadrupole Time-of-Flight Mass Spectrometry: A Powerful Tool for Proteomics Research. Anal. Chem. 2000, 72, 2132-2141.

22. Zhan, Y.; Richardson, B.; Becklin, R.; Savage, J. K. B.; Peltier, J. Development of an LC-MALDI Method for the Analysis of Protein Complexes. J. Am. Chem. Soc. 2004, 15, 803-822

23. Yergey, A.; Coorssen, J.; Backlund, P.; Blank, P.; Humphrey, G.; Campbell, J.; Vestal, M.; De Novo Sequencing of Peptides Using MALDI/ TOF-TOF. J. Am. Soc. Mass Spectrom. 2002, 13, 784-791.

24. Campbell, J. In Mapping the Properties of Center of Mass Collision Energy on a MALDI TOF/TOF Mass Spectrometer-Fundamentals and Applications,
Proceedings of the 51st ASMS Conference on Mass Spectrometry and Allied Topics, Montreal, Quebec, 2003.

25. Karas, M.; Kruger, R. Ion Formation in MALDI: The Cluster Ionization Mechanism. Chem. Rev. 2003, 103, 427-439.

26. Berkenkamp, S.; Menzel, C.; Hillenkamp, F.; Dreisewerd, K. Measurements of Mean Initial Velocities of Analyte and Matrix Ions in Infrared Matrix-assisted Laser Desorption Ionization Mass Spectrometry. J. Am. Soc. Mass Spectrom. 2002, 13, 209-220.

27. Menzel, C.; Dreisewerd, K.; Berkenkamp, S.; Hillenkamp, F. The Role of the Laser Pulse Duration in Infrared Matrix-Assisted Laser Desorption/ Ionization Mass Spectrometry. J. Am. Soc. Mass Spectrom. 2002, 13, 975-984.

28. Dreisewerd, K. The Desorption Process in MALDI. Chem. Rev. 2003, 103, 395- 425 .

29. Stevenson, E.; Breuker, K.; Zenobi, R. Internal Energies of Analyte Ions Generated from Different Matrix-assisted Laser Desorption/Ionization Matrices. J. Mass Spectrom. 2000, 35, 1035-1041.

30. Gabelica, V.; Schulz, E.; Karas, M. Internal Energy Build-up in Matrix-assisted Laser Desorption/Ionization. Int. J. Mass Spectrom. 2004, 39, 579-593.

31. Bencsura, A.; Navale, V.; Sadeghi, M.; Vertes, A. Matrix-Guest Energy Transfer in Matrix-assisted Laser Desorption. Rapid Commun. Mass Spectrom. 1997, 11, 679-682.

32. Sadeghi, M.; Wu, X.; Vertes, A. Conformation Changes, Complexation, and Phase Transition in Matrix-Assisted Laser Desorption. J. Phys. Chem. B. 2001, 105, 2578-2587.

33. Luo, G.; Marginean, I.; Vertes, A. Internal Energy of Ions Generated by Matrix-assisted Laser Desorption/Ionization. Anal. Chem. 2002, 74 6185-6190.

34. Moskovets, E.; Vertes, A. Fast Dynamics of Ionization in Ultraviolet Matrix-assisted Laser Desorption Ionization of Biomolecules. J. Phys. Chem. B. 2002, 106, 3301-3306.

35. Zhigilei, L.; Kodali, P.; Garrison, B. Molecular Dynamics Model for Laser Ablation and Desorption of Organic Solids. J. Phys. Chem. B. 1997, 101, 2028-2037

36. Zhigilei, L.; Kodali, P.; Garrison, B. On the Threshold Behavior in Laser Ablation of Organic Solids. Chem. Phys. Lett. 1997, 276, 269-273.

37. Zhigilei, L. Garrison, B. Velocity Distributions of Analyte Molecules in Matrix-assisted Laser Desorption from Computer Simulations. Rapid Commun. Mass Spectrom 1998, 12, 1273-1277.

38. Zhigilei, L.; Kodali, P.; Garrison, B. A Microscopic View of Laser Ablation. J. Phys. Chem. B. 1998, 102, 2845-2853.

39. Knochenmuss, R.; Zhigilei, L. Molecular Dynamics Model of Ultraviolet Matrix-assisted Laser Desorption/Ionization Including Ionization Processes. J. Phys. Chem. B. 2005, 109, 22947-22957.

40. Knochenmuss, R. A Quantitative Model of Ultraviolet Matrix-assisted Laser Desorption/Ionization. J. Mass Spectrom. 2002, 37, 867-877.

41. Liu, Z.; Schey, K. Optimization of a MALDI TOF-TOF Mass Spectrometer for Intact Protein Analysis. J. Am. Soc. Mass Spectrom. 2005, 16, 482-490.

42. Brown, R.; Lennon, J. Sequence-specific Fragmentation of Matrixassisted Laser Desorbed Protein/Peptide Ions. Anal. Chem. 1995, 65, 3990-3999.

43. Takayama, M. In Source Decay Characteristics of Peptides in Matrix Assisted Laser Desorption/Ionization Time-of-Flight Mass Spectrometry. J. Am. Soc. Mass Spectrom. 2001, 12, 420-427.

44. Meot-Ner (Mautner), M.; Dongre, A.; Somogyi, A.; Wysocki, V. Thermal Decomposition Kinetics of Protonated Peptides and Peptide Dimers, and Comparison with Surface-Induced Dissociation. Rapid Commun. Mass Spectrom. 1995, 9, 829-836.

45. Speir, J.; Amster, I. An Investigation of the Energetics of Peptide Ion Dissociation by Laser Desorption Chemical Ionization Fourier Transform Mass Spectrometry. J. Am. Soc. Mass Spectrom. 1995, 6, 1069-1078.

46. Dongre, A.; Somogyi, A.; Wysocki, V. Surface-induced Dissociation: An Effective Tool to Probe Structure, Energetics and Fragmentation Mechanisms of Protonated Peptides. J. Mass Spectrom. 1996, 31, 339-350.

47. Schnier, P.; Price, W.; Strittmatter, E.; Williams, E. Dissociation Energetics and Mechanisms of Leucine Enkephalin $(\mathrm{M}+\mathrm{H})+$ and $(2 \mathrm{M}+\mathrm{X})+$ Ions $(\mathrm{X}=\mathrm{H}, \mathrm{Li}, \mathrm{Na}, \mathrm{K}$ and $\mathrm{Rb}$ ) Measured by Blackbody Infrared Radiative Dissociation. J. Am. Soc. Mass Spectrom. 1997, 8, 771-780.

48. Hager, J. Product Ion Spectral Simplification Using Time-delayed Fragment Ion Capture with Tandem Linear Ion Traps. Rapid Commun. Mass Spectrom. 2003, 17, 1389-1398.

49. Zhu, X.; Papayanopoulos, I. Improvement in the Detection of Low Concentration Protein Digests on a MALDI TOF/TOF Workstation by Reducing $\alpha$-Cyano-4-hydroxycinnamic Acid Adduct Ions. J. Biomol. Tech. 2003, 14, 298-307.

50. Smirnov, I.; Zhu, X.; Taylor, T.; Huang, Y.; Ross, P.; Papayanopoulos, I.; Martin, S.; Pappin, D. Suppression of $\alpha$-Cyano-4-hydroxycinnamic Acid Matrix Clusters and Reduction of Chemical Noise in MALDI-TOF Mass Spectrometry. Anal. Chem. 2004, 76, 2958-2965.

51. Rakov, V.; Borisov, O.; Whitehouse, C. Establishing Low-energy Sequential Decomposition Pathways of Leucine Enkephalin and Its Nand C-Terminus Fragments Using Multiple-Resonance CID in Quadrupolar Ion Guide. J. Am. Soc. Mass Spectrom. 2004, 15, 1794-1809.

52. Knochenmuss, R. Photoionization Pathways and Free Electrons in UV-MALDI. Anal. Chem. 2004, 76, 3179-3184.

53. Knochenmuss, R. Ion Formation Mechanisms in UV-MALDI. Analyst 2006, 131, 966-986.

54. Stein, S. Personal communication.

55. Knochenmuss, R.; Zenobi, R. MALDI Ionization: The Role of In-Plume Processes. Chem. Rev. 2003, 103, 441-452. 